\title{
Screening for Impaired Cognitive Domains in a Large Parkinson's Disease Population and Its Application to the Diagnostic Procedure for Parkinson's Disease Dementia
}

\author{
Kouichi Ohta $^{a}$ Kazushi Takahashib ${ }^{\text {J Ju Gotoh }}{ }^{c}$ Keiji Yamaguchid \\ Morinobu Seki ${ }^{e}$ Yoshihiro Nihei ${ }^{e}$ Satoko Iwasawa ${ }^{f}$ \\ Norihiro Suzuki ${ }^{\text {e }}$ on behalf of the Keio Parkinson's Disease Database \\ a Department of Neurology, Tachikawa Hospital, Tachikawa, ${ }^{b}$ Department of Neurology, \\ Tokyo Metropolitan Neurological Hospital, Fuchu, 'Department of Neurology, Saiseikai \\ Yokohamashi Tobu Hospital, Yokohama, ${ }^{\mathrm{d}}$ Department of Neurology, Mito Red Cross \\ Hospital, Mito, and Departments of e Neurology and ${ }^{f}$ Preventive Medicine and Public \\ Health, Keio University School of Medicine, Shinjuku, Japan
}

\section{Key Words}

Parkinson's disease - Dementia - Cognitive domain - Executive function - Mini-Mental State Examination · Montreal Cognitive Assessment

\section{Abstract}

Background: Dementia is a new focus of research on improved treatment for Parkinson's disease (PD). In 2007, a screening tool for PD dementia (PD-D) was developed by the Movement Disorder Society (Level I testing), which still requires verification by a large population study. Methods: We conducted a cross-sectional and multicenter study including 13 institutions administering the Mini-Mental State Examination (MMSE) and Montreal Cognitive Assessment (MoCA) to 304 PD patients (mean age: $70.6 \pm 8.3$ years; mean Hoehn and Yahr stage: $2.7 \pm$ 0.7). Results: In all, $34.5 \%$ of the patients had MMSE scores $<26 ; 94.3 \%$ of these patients had impairments in $\geq 2$ cognitive domains and met the criteria for probable PD-D by Level I testing. Executive dysfunction combined with attention and memory impairment was most common (51.4\%). In the Level I subtests of executive function, the score for phonemic fluency declined by $<50 \%$ in patients with high MoCA scores (24-30 points) and lacked specificity for PD-D. No patient had visuospatial impairment (measured by the pentagon copying subtest) alone, and the score for pentagon copying stayed at $\geq 70 \%$ even in patients with low MMSE scores (12-25 points), therefore lacking sensitivity for PD-D. Conclusions: Level I testing with administration of the MMSE and MoCA is a practical and efficient screening tool for PD-D. However, the phonemic fluency and pentagon copying tests should be replaced by more specific/sensitive ones when screening for PD-D. 


\section{Introduction}

Nondopaminergic and nonmotor symptoms of Parkinson's disease (PD), which include psychiatric, autonomic, and gastrointestinal symptoms, are sometimes present before a diagnosis of PD and almost inevitably emerge with the disease progression [1]. In contrast to motor symptoms of PD, nonmotor symptoms have been poorly recognized and inadequately treated. However, methods for recognizing and quantifying the nonmotor symptoms of PD have become a new focus of research that will form the basis for improved treatments [1].

Among the nonmotor symptoms, cognitive impairment is of utmost interest. In PD patients, cognitive impairment adversely influences their activities of daily living and quality of life [2] and worsens caregiver burden [3]. A community-based study found that within a group of patients with a diagnosis of PD of at least 20 years, $83 \%$ had experienced dementia, and a similar number of patients had experienced frequent falls (87\%), moderate dysarthria (81\%), visual hallucinations (74\%), or urinary incontinence (71\%) [4]. In PD dementia (PD-D) patients, $\alpha$-synuclein pathology can be widespread in the cerebral cortex [5]. In addition, there is less acetylcholinesterase activity in the cerebral cortex of PD-D patients than of Alzheimer's disease patients [6]. Moreover, clinical studies have reported on the effectiveness of cholinesterase inhibitors for treating cognitive impairment in PD-D patients [7-9].

The prevalence of cognitive dysfunction among PD patients has led to the recognition that a diagnostic criterion is needed for PD-D. As a result, the Movement Disorder Society Task Force published diagnostic criteria for PD-D in 2007 [10] and simultaneously proposed a practical diagnostic procedure for PD-D with the aim of its widespread international application [11]. The diagnostic procedure comprises Level I and Level II testing. Level I testing includes a simple and short algorithm based on current cognitive tools that can be used in an office or at the bedside. It is considered a screening tool for diagnosing PD-D, whereas Level II testing provides a more detailed assessment that characterizes the components of PD-D and monitors the elements that may be responsive to intervention.

Level I testing was designed as a screening tool for use in the clinic and in clinical trials. Although it has been used in practice [12], no study has yet verified its validity or utility in a large sample of PD patients. In this study, we used Level I testing on 304 PD patients with the objective of determining which patients would be diagnosed with PD-D and what impairments would be diagnosed among the 4 cognitive domains tested.

\section{Subjects and Methods}

\section{Subjects}

The subjects included in our study were Japanese patients with idiopathic PD who were clinically diagnosed and treated by board-certified neurologists at 1 of the 13 participating institutions between July and September 2011. A diagnosis of PD was made according to the UK Parkinson's Disease Society Brain Bank criteria [12] as well as a differential diagnosis. The study design and protocol were approved by the ethics committees for human research of the Keio University School of Medicine and all the participating institutions. All subjects agreed to participate in this study and provided their informed consent.

\section{Assessments of Clinical and Neurological Features}

This was a cross-sectional study which examined the clinical and neurological features of these PD patients, including sex, age at the assessment, education, disease duration, and the Hoehn and Yahr (H\&Y) stage. All patients were examined in the 'on' state with respect to 
Table 1. Algorithm for diagnosing PD-D at Level I (Level I testing) [11]

1 A diagnosis of PD based on the UK Parkinson's Disease Society Brain Bank criteria [12]

2 PD developed prior to the onset of dementia

3 An MMSE [23] score <26

4 Cognitive deficits severe enough to affect daily living (caregiver interview or Pill Questionnaire)

5 Impairment in $\geq 2$ of the following tests:

- Months reversed or seven backward (attention) ${ }^{1}$

- Lexical fluency or clock drawing (executive function) ${ }^{1}$

- MMSE pentagons (visuospatial function) ${ }^{1}$

- Three-word recall (memory) ${ }^{1}$

For a diagnosis of probable PD-D, all the criteria in this table must be fulfilled. The presence of apathy, depressed mood, delusions, or excessive daytime sleepiness may support the diagnosis of probable PD-D. The presence of major depression, delirium, or any other abnormality that may by itself cause significant cognitive impairment makes the diagnosis uncertain.

${ }^{1}$ Relevant cognitive domain.

dopaminergic medication. Their cognitive function was evaluated with the Japanese versions of the Mini-Mental State Examination (MMSE) [13] and Montreal Cognitive Assessment (MoCA) [14]. The MMSE consists of 10 subtests scored to a total of 30 points: pentagon copying ( 1 point), serial 7 subtraction ( 5 points), immediate ( 3 points) and delayed 3 -word recall ( 3 points), naming ( 2 points), repeating a sentence ( 1 point), 3-stage commands ( 3 point), reading a sentence ( 1 point), writing a sentence ( 1 point), and orientation to time and place (10 points). The MoCA consists of 12 subtests also scored to a total of 30 points: cube copying (1 point), clock drawing ( 3 points), trail making ( 1 point), phonemic fluency (1 point), verbal abstraction ( 2 points), digit span ( 2 points), serial 7 subtraction ( 3 points), target tapping ( 1 point), delayed 5 -word recall ( 5 points), naming ( 3 points), repeating 2 sentences ( 2 points), and orientation to time and place (6 points).

\section{Algorithm for Level I Testing}

We used the algorithm for Level I testing to examine the present patient population (table 1) [11]. In this algorithm, the diagnosis of PD-D is based on the following 5 criteria: (1) diagnosis of PD, (2) PD developed prior to the onset of dementia, (3) a decreased global cognitive efficiency, (4) a cognitive deficiency that impairs daily life, and (5) impairment in $>1$ cognitive domain.

In this study, however, we did not investigate whether cognitive deficits were severe enough to affect daily living (item 4, typically assessed with a caregiver interview or the Pill Questionnaire). For item 5, the results of the following subtests of the MoCA and MMSE were used: serial 7 subtraction ('seven backward' in table 1), phonemic fluency ('lexical fluency'), and clock drawing from the MoCA, and pentagon copying ('MMSE pentagons') and delayed 3 -word recall from the MMSE. Their relevant cognitive domains were designated as 'attention' for serial 7 subtraction, 'executive function' for phonemic fluency and clock drawing, 'visuospatial function' for pentagon copying, and 'memory' for delayed 3-word recall (table 1) [11].

In the months reversed test, the patient must name the months in reverse order. However, Japanese months are named by number, which is different from their naming in English; thus, the months reversed test was excluded from this study. According to the Level I testing procedures [11], test results that indicated impairment were: $\geq 2$ incorrect responses on the seven backward test, a score of $\leq 9$ words in the lexical fluency test, an inability to correctly draw the clock face or clock hands pointing to the correct time in the clock drawing test, an inability 
Table 2. Comparison of clinical/neurological features between 3 groups based on their MoCA score

\begin{tabular}{lrrr}
\hline & $\begin{array}{l}\text { Low score } \\
(\mathrm{n}=98)\end{array}$ & $\begin{array}{l}\text { Middle score } \\
(\mathrm{n}=98)\end{array}$ & $\begin{array}{l}\text { High score } \\
(\mathrm{n}=108)\end{array}$ \\
\hline Age, years & $74.3 \pm 6.6$ & $72.0 \pm 7.7$ & $66.0 \pm 8.1$ \\
Education, years & $11.4 \pm 3.2$ & $12.3 \pm 2.7$ & $13.2 \pm 2.3$ \\
Disease duration, years & $6.7 \pm 5.1$ & $7.0 \pm 5.9$ & $6.1 \pm 4.3$ \\
H\&Y stage & $3.0 \pm 0.8$ & $2.7 \pm 0.7$ & $2.5 \pm 0.6$ \\
\hline
\end{tabular}

Data are means \pm SD. Low score $=5-18$; middle score $=19-23$; high score $=24-30$.

Table 3. Comparison of clinical/neurological features between 3 groups based on their MMSE score

\begin{tabular}{lccc}
\hline & $\begin{array}{l}\text { Low score } \\
(\mathrm{n}=105)\end{array}$ & $\begin{array}{l}\text { Middle score } \\
(\mathrm{n}=93)\end{array}$ & $\begin{array}{l}\text { High score } \\
(\mathrm{n}=106)\end{array}$ \\
\hline Age, years & $73.9 \pm 7.1$ & $71.0 \pm 7.4$ & $67.1 \pm 8.7$ \\
Education, years & $11.6 \pm 3.2$ & $12.3 \pm 2.7$ & $13.2 \pm 2.3$ \\
Disease duration, years & $6.5 \pm 5.0$ & $7.5 \pm 5.8$ & $5.8 \pm 4.5$ \\
H\&Y stage & $2.9 \pm 0.8$ & $2.7 \pm 0.7$ & $2.6 \pm 0.6$ \\
\hline
\end{tabular}

Data are means \pm SD. Low score $=12-25$; middle score $=26-28$; high score $=29-30$.

to correctly draw 2 pentagons that overlap in the pentagon copying test, and $\geq 1$ word missing in the 3-word recall test.

\section{Statistical Analysis}

The JMP ${ }^{\circledR}$ software version 8.0 (SAS Institute, Tokyo, Japan) was used for all statistical analyses. The level of statistical significance was defined as $p<0.05$. A one-way ANOVA and the Tukey-Kramer HSD test were used to examine differences among groups.

\section{Results}

\section{Subject Demographics}

A detailed description of the demographics of the 304 PD patients included in our study has been reported elsewhere [15]. Briefly, the subjects were 166 men and 138 women with a mean age \pm SD of $70.6 \pm 8.3$ (range: $42-90$ ) years; their education lasted $12.5 \pm 2.6$ (range: $1-20$ ) years, the disease duration was $6.6 \pm 5.1$ (range: $0.2-30.1$ ) years, and their H\&Y stage was $2.7 \pm 0.7$ (range: $1-5$ ).

\section{Differences among the MoCA and MMSE Tests}

The subjects were grouped into tertiles according to their total MoCA score (table 2). Similarly, the subjects were grouped into tertiles according to their total MMSE score (table 3). The scores for the 12 MoCA subtests are presented by group in figure 1a. The single subtest scores decreased significantly as the total MoCA scores decreased from the high-MoCA-score group to the middle- and low-MoCA-score groups $(\mathrm{p}<0.05)$. Exceptionally, the decreases in 


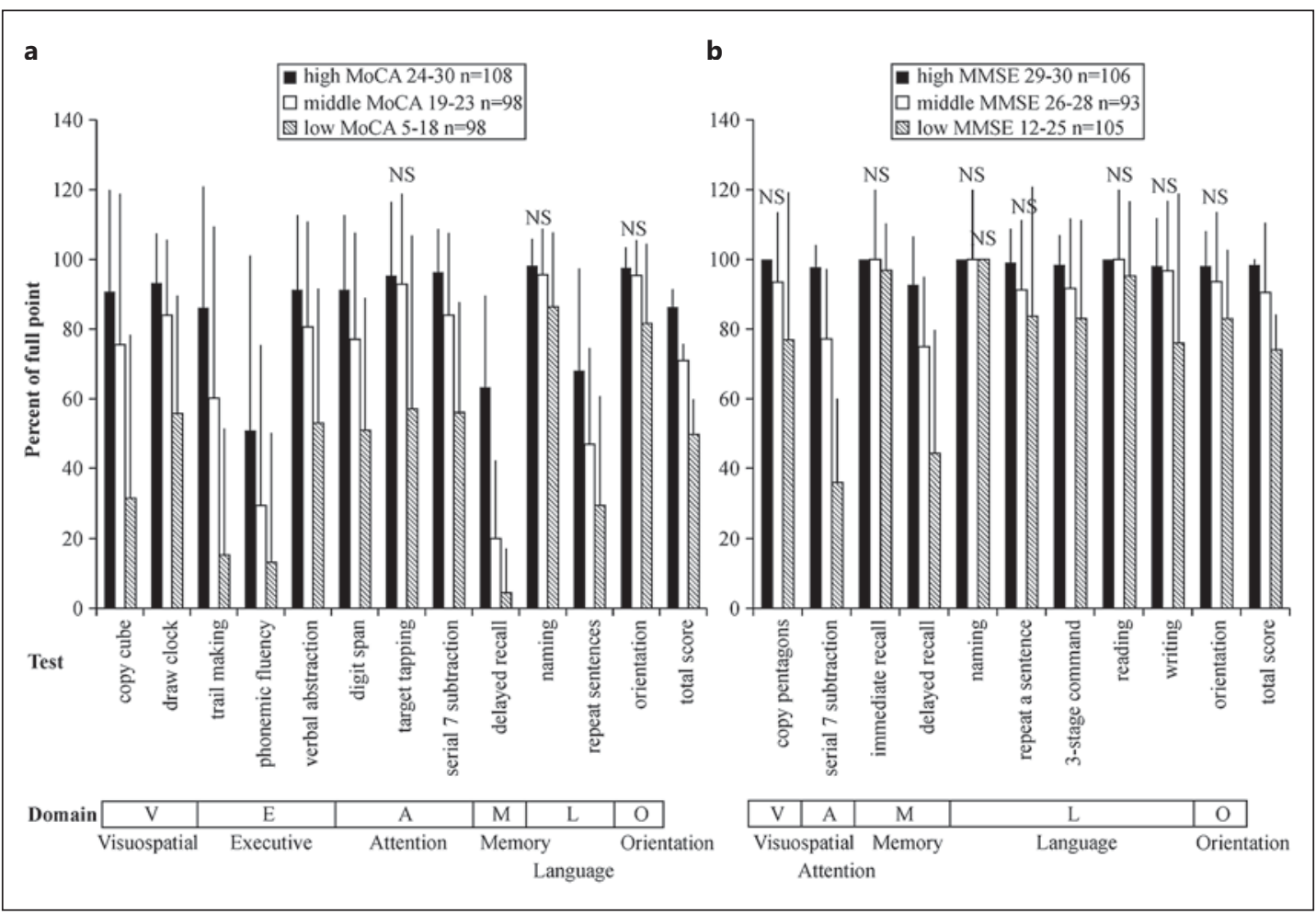

Fig. 1. Scores for the 12 subtests of the MoCA (a) and the 10 subtests of the MMSE (b) in tertile groups of PD patients $(n=304)$ according to their total MoCA or MMSE scores. Scores are shown as mean percentages \pm SD of each full point. Lower panel: cognitive domains relevant to each test $[17,23]$. The subtest scores decreased significantly as the total MoCA score decreased from the high-MoCA-score group to the middle- and low-MoCA-score groups ( $\mathrm{p}<0.05$ ), except for the columns marked 'NS' (not significant).

scores for the naming, target tapping, and orientation tests from the high- to the middleMoCA-score group were not significantly different. Therefore, the decline in the total MoCA score mirrors a decline in the scores for almost all the subtests.

The differences between the 12 MoCA subtests are presented in figure 2a. Naming and orientation were preserved ( $>80 \%)$ even in the low-MoCA-score group. In contrast, sentence repeating, phonemic fluency, and delayed recall scores decreased remarkably $(<70 \%)$ even in the high-MoCA-score group. Trail making was preserved in the high-MoCA-score group but decreased steeply in the middle- and low-MoCA-score groups.

The scores for the 10 MMSE subtests are presented by group in figure $1 \mathrm{~b}$. For most of the subtests ( 7 of 10), the scores failed to show a significant decrease from the high- to the middleMMSE-score group, and the scores for the naming subtest failed to decrease even in the lowMMSE-score group. All domains were preserved ( $>90 \%)$ in the high-MMSE-score group, indicating that no MMSE subtest was significative for these PD patients (fig. 2b). This finding is quite different from those obtained for the MoCA. A significant decrease was observed only for the serial 7 subtraction and delayed recall subtests in the low-MMSE-score group $(<50 \%$, $\mathrm{p}<0.05$ ).

We observed differences between the MoCA and MMSE subtests that measured similar abilities. For example, the MoCA includes a 5-word delayed recall test, whereas the MMSE provides a 3-word delayed recall test; 5-word recall according to the MoCA was $<70 \%$ even in the high-MoCA-score group, whereas 3-word recall according to the MMSE was relatively 


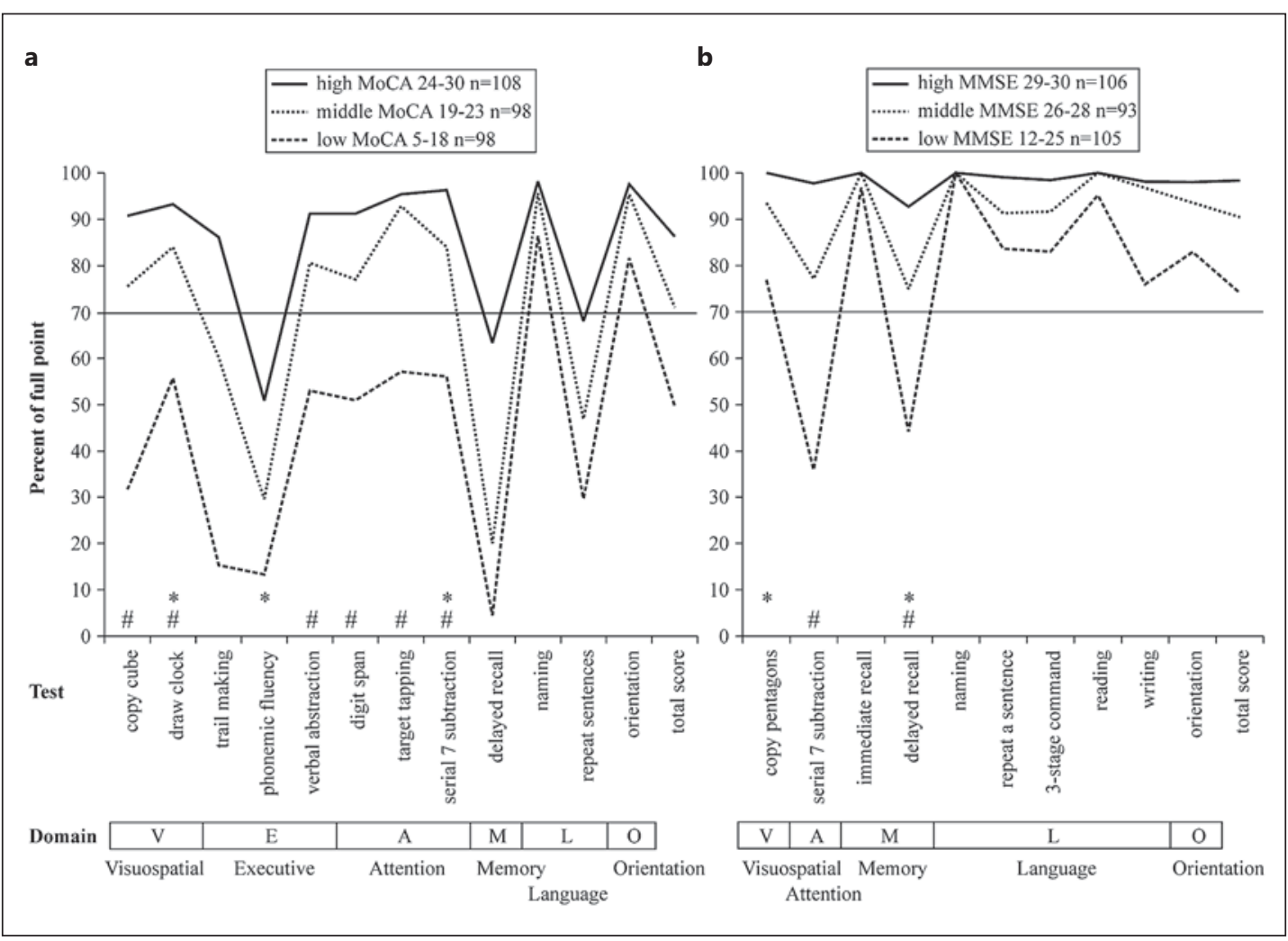

Fig. 2. Scores for the 12 subtests of the MoCA (a) and the 10 subtests of the MMSE (b) in tertile groups of PD patients $(n=304)$ according to their total MoCA or MMSE scores. Scores are shown as mean percentages of each full point. Lower panel: cognitive domains relevant to each test $[17,23] . *$ Test quoted in Level I testing for PD-D [11]. \# Test score which was $<70 \%$ only in the low-score group but not in the high- or middle-score groups.

preserved (fig. 1, 2). In addition, the MoCA includes a subtest in which 2 sentences are repeated, whereas the MMSE includes a subtest in which 1 sentence is repeated. Furthermore, the length and complexity of the sentences are greater in the MoCA. The repeating 2 sentences subtest of the MoCA showed a score $<70 \%$ in the high-MoCA-score group, but the score for the repeating a sentence subtest of the MMSE was preserved even in the low-MMSE-score group.

\section{Application of Diagnostic Criteria for PD-D Screening (Level I Testing)}

Items 1 (diagnosis of PD) and 2 (dementia preceding PD) in Level I testing (table 1) were applicable to the subjects participating in this study [11]. For item 3 (decreased global cognitive efficiency), 105 subjects (34.5\%) showed an MMSE score $<26$. With respect to item 5 (dysfunction in $>1$ cognitive domain), we observed that 53 subjects $(17.4 \%$ ) showed no impairment in any cognitive domain, whereas the remaining subjects showed impairments in $1(\mathrm{n}=74 ; 24.3 \%), 2(\mathrm{n}=87 ; 28.6 \%), 3(\mathrm{n}=74 ; 24.3 \%)$, or $4(\mathrm{n}=16 ; 5.3 \%)$ cognitive domains. Therefore, 177 subjects (58.2\%) showed impairments in $\geq 2$ cognitive domains. Among these 177 subjects, 99 also had MMSE scores $<26$ (32.6\% of all subjects; $94.3 \%$ of the total group with MMSE scores $<26$ ) and therefore met the diagnostic criteria for PD-D [11]. Only 6 subjects (5.7\% of 105 subjects) with MMSE scores $<26$ did not meet the diagnostic criteria for PD-D, because they had only 1 impaired cognitive domain. Of the 105 
Fig. 3. Numbers of PD patients with 0-4 impaired cognitive domains according to Level I testing for PD-D ( $\mathrm{n}=304)$ [11]. Data are shown for 2 groups according to their total MMSE scores (cutoff: 26 points).

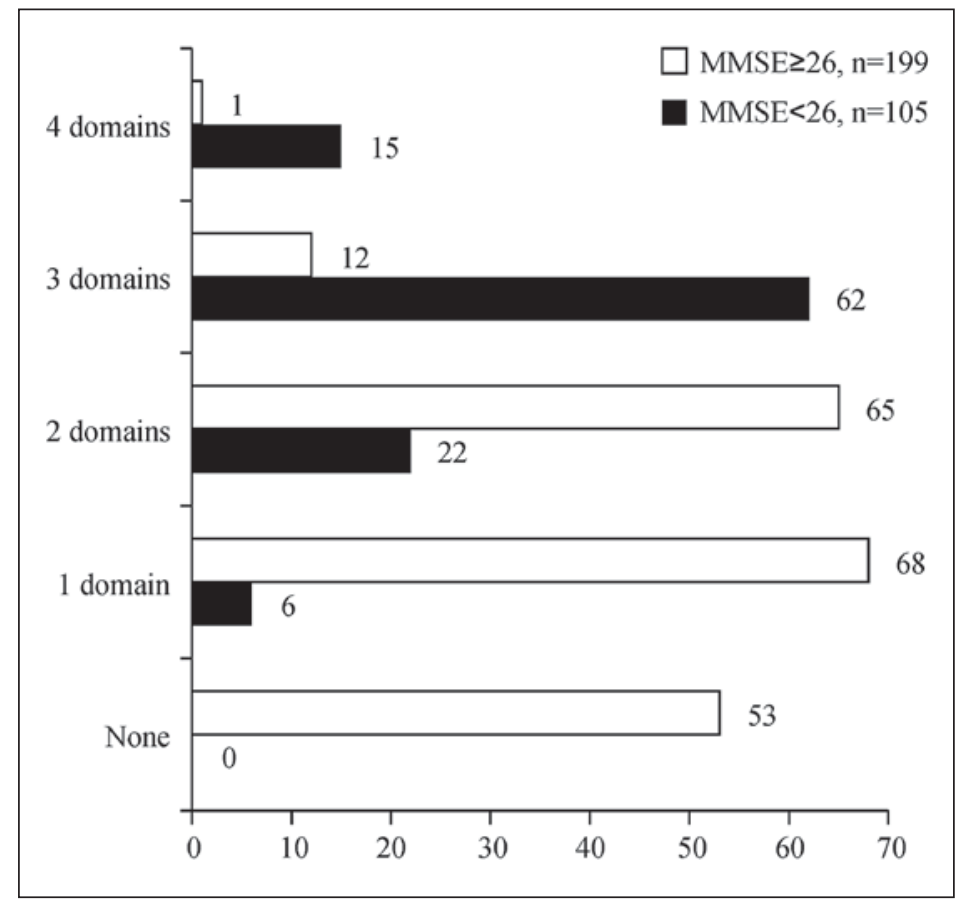

subjects with MMSE scores $<26$, most showed impairments in 3 cognitive domains ( $\mathrm{n}=62$; $59.0 \%)$.

Figure 3 shows the number of subjects with MMSE scores $<26$ or $\geq 26$, along with the number of impaired domains. No subject with an MMSE score $<26$ was without impairment in any domain. Among the subjects with MMSE scores $\geq 26(n=199)$, we observed $1(n=68$; $34.2 \%), 2(n=65 ; 32.7 \%), 3(n=12 ; 6.0 \%)$, and $4(n=1 ; 0.5 \%)$ impaired domains; $53(26.6 \%)$ had no impairment in any cognitive domain.

Figure 4 shows the proportions of subjects with MMSE scores $<26$ or $\geq 26$, along with the number of impaired domains. Subjects with MMSE scores $\geq 26$ accounted for the majority of subjects with $\leq 1$ impaired domain, and they accounted for $74.7 \%$ of subjects with 2 impaired domains. In contrast, subjects with MMSE scores $<26$ accounted for the majority of subjects with 3 or 4 impaired domains. This finding was as expected because patients with lower MMSE scores should have an increased likelihood of being affected in more cognitive domains.

Figure 5a shows the numbers of subjects with impairments for each of the 4 cognitive domains. The most common impairment was executive dysfunction (224 subjects; $73.7 \%$ ), followed by memory $(\mathrm{n}=161 ; 53.0 \%)$ and attention $(\mathrm{n}=119 ; 39.1 \%)$ impairments. Visuospatial functional impairments were the least common and were apparent in only 30 subjects (9.9\%). Of the 224 subjects who showed executive dysfunction, most exhibited impairment in lexical fluency (180 subjects; $80.4 \%)$ or clock drawing ( $n=133 ; 59.4 \%)$, whereas 89 subjects (39.7\%) displayed impairments in both (fig. 5b).

Figure 6 shows the combinations of impaired cognitive domains observed. The most common combinations in the group with MMSE scores $\geq 26$ were executive dysfunction alone (49 subjects; $24.6 \%$ ) and executive dysfunction with memory impairment ( $\mathrm{n}=47$; $23.6 \%)$, followed by executive dysfunction with attention impairment ( $\mathrm{n}=16 ; 9.5 \%)$ and memory impairment alone $(n=15 ; 7.5 \%)$. The most common combination of impaired cognitive domains in the group with MMSE scores $<26$ was executive dysfunction with both memory and attention impairments $(n=54 ; 51.4 \%)$, followed by impairments in all 4 
Dementia

and Geriatric

Cognitive Disorders

Fig. 4. Percentages of PD patients with 0-4 impaired cognitive domains according to Level I testing for PD-D ( $n=304)$ [11]. Data are shown for 2 groups according to their total MMSE scores (cutoff: 26 points).

Fig. 5. a Numbers of PD patients with impairments according to Level I testing for PD-D ( $\mathrm{n}=304)$ [11], listed for each cognitive domain. b Total number of PD patients with executive dysfunction, as well as the number of patients with impairments in clock drawing or phonemic fluency or both.

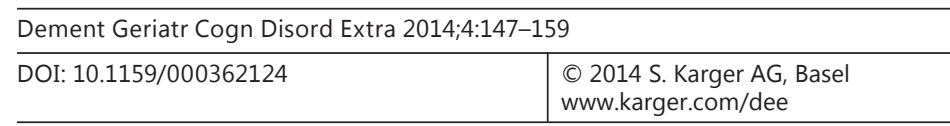

Ohta et al.: Screening Cognitive Domains in Parkinson's Disease
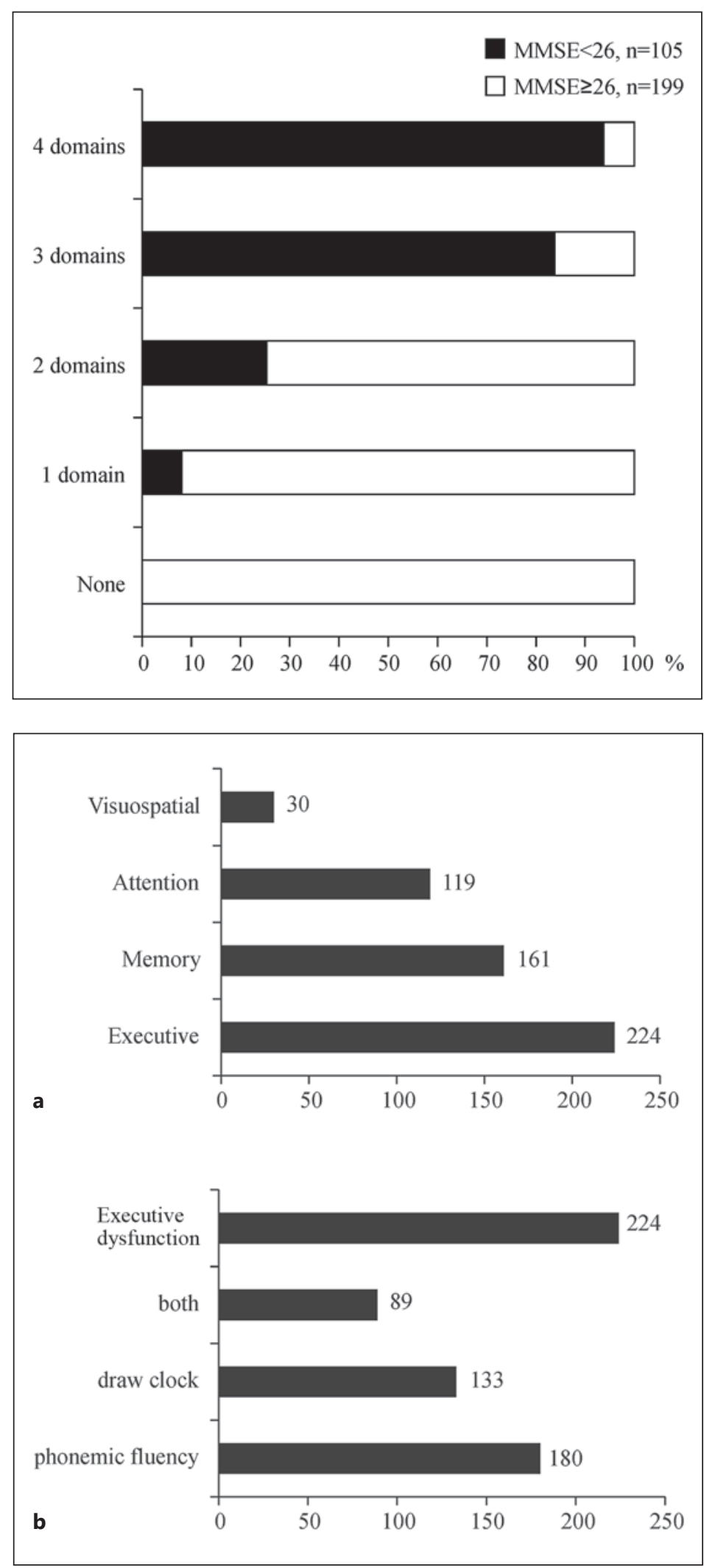
Fig. 6. Numbers of PD patients with different combinations of impaired cognitive domains according to Level I testing for PD-D $(\mathrm{n}=304)$ [11]. $\mathrm{E}=$ Executive function; $\mathrm{M}=$ memory; $\mathrm{A}=$ attention; $\mathrm{V}=$ visuospatial function.

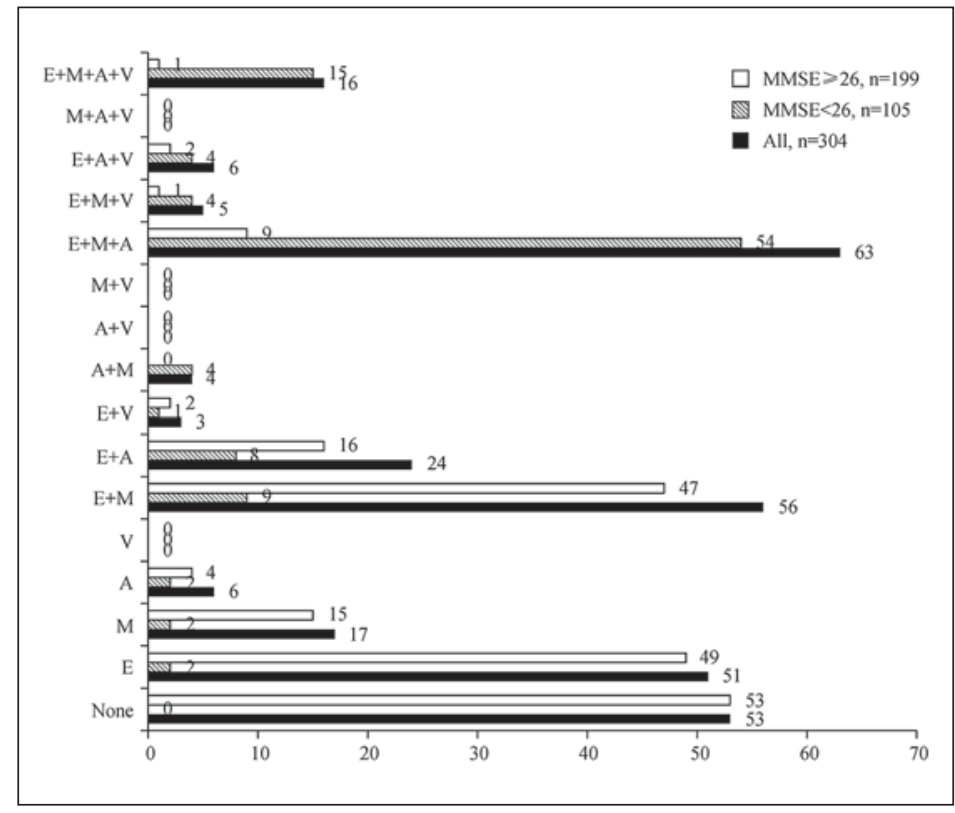

domains $(n=15 ; 14.3 \%)$. Interestingly, visuospatial impairment was always accompanied by executive dysfunction; however, visuospatial impairment alone, or in combination with attention, memory, or both attention and memory impairments, did not occur in any of the subjects.

\section{Possible Alternatives to Level I Testing}

For the purpose of screening PD patients for dementia, it is necessary to use a test in which achievement does not decline in PD patients who have normal global cognitive efficiency (as measured by the total MMSE or MoCA score) but declines in those patients with impaired global cognitive efficiency indicative of dementia. With regard to the hypothesis that an appropriate test is one in which the high- and middle-total-score groups by the MMSE or the MoCA maintain a score $\geq 70 \%$ while the low-total-score group scores $<70 \%$, the copy cube, draw clock, verbal abstraction, digit span, target tapping, and serial 7 subtraction tests of the MoCA (marked \# in fig. 2a) and the serial 7 subtraction and 3-word delayed recall tests of the MMSE (marked \# in fig. 2b) are considered appropriate. In Level I testing, this includes the clock drawing, serial 7 subtraction, and delayed 3-word recall subtests, but not the phonemic fluency or pentagon copying subtests.

As regards evaluating executive function, scores for the phonemic fluency test declined markedly to approximately 50\% in the high-MoCA-score group, whose scores (24-30) were consistent with PD without dementia [16]. This suggests that it may lack specificity for dementia screening. The other subtest that evaluates executive function in Level I testing is the clock drawing test. Scores for this test declined to $<70 \%$ only in the low-MoCA-score group. This suggests the clock drawing test may be more appropriate than the phonemic fluency test for screening for a decline in executive function in PD-D.

On the other hand, when evaluating visuospatial function, scores for the pentagon copying test stayed at $\geq 70 \%$ even in the low-MMSE-score group, whose scores (12-25) were below the 26-point cutoff for PD-D [11]. This suggests that it may lack sensitivity for dementia when screening PD patients. No alternative to the pentagon copying test is given in Level I testing. The authors of the original MoCA test indicated that the cube copying and clock drawing tests 
are concerned with visuospatial function [17], but in Level I testing, the clock drawing test is used for executive function. For the cube copying test, the authors stated that if any of the following criteria are not met, the abnormal result was assigned: (1) drawing must be 3 dimensional, (2) all lines are drawn, (3) no line is added, (4) lines are relatively parallel and their length is similar' [17]. Applied to the 304 subjects in the present study, 101 were classified as abnormal according to the cube copying test, including 43 subjects in the group with MMSE scores $\geq 26$ and 58 subjects in the group with MMSE scores $<26$. These are far more than the 30 subjects with abnormal results for the pentagon copying test, and almost as many as the 119 subjects who were categorized as abnormal in attention by the serial 7 subtraction test.

\section{Discussion}

Some previous studies have examined the screening properties of Level I testing for PD-D [11]. Martinez-Martin et al. [18] have demonstrated that Level I testing is more sensitive for the detection of PD-D, detecting up to 22\% more cases than the DSM-IV. Di Battista et al. [19] validated Level I testing in comparison with the DSM-IV criteria for PD-D and revealed its relatively lower sensitivity (78\%) but high specificity (95.5\%) with a 12\% prevalence of PD-D in 76 PD patients. The authors raised their doubts about the sensitivity of an MMSE cutoff value of 26 , stating this to be the main cause of false negatives, and also pointed out that more sensitive cognitive domain-related psychometric tests would maximize the screening property of Level I testing. Barton et al. [20] compared Level I testing with full neuropsychological testing for detecting PD-D in 91 PD patients and found that $7.7 \%$ of the subjects met the Level I testing criteria, while $16.5 \%$ of the subjects were classified as PD-D by full neuropsychological testing. They revealed that Level I testing showed $100 \%$ specificity but only $46.7 \%$ sensitivity for diagnosing PD-D compared with the full neuropsychological assessment. They showed that the low sensitivity is largely due to the total MMSE cutoff values for decreased global cognitive efficiency and the 15-item Geriatric Depression Scale score for excluding depression.

We found that $34.5 \%$ of all PD patients examined in the present study had MMSE scores $<26$. Most of these patients (94.3\%) were impaired in $\geq 2$ cognitive domains and therefore were cases of probable PD-D. These patients accounted for $32.6 \%$ of the total number of PD patients. Many of these patients (59\%) showed impairment in 3 cognitive domains, and patients rarely showed impairment in a single cognitive domain. At least 1 of these 4 cognitive domains was always impaired in PD patients with MMSE scores $<26$. Even among PD patients with MMSE scores $\geq 26$, many had impairments in 1 or 2 cognitive domains, and the proportion of those with no impairment in any cognitive domain was $26.6 \%$. Subjects with MMSE scores $\geq 26$ accounted for $75 \%$ of PD patients who had impairments in 2 cognitive domains, which was far more than in the group with MMSE scores $<26$.

Among the cognitive domains, executive function, which was tested by the phonemic fluency and clock drawing subtests, was most frequently impaired; visuospatial function, which was tested by the pentagon copying test, was least frequently impaired. No patient showed visuospatial impairment alone, and an impaired visuospatial function was always accompanied by executive dysfunction. Among the subjects in the group with MMSE scores $\geq 26$, the most common combinations were executive dysfunction alone or executive dysfunction with memory impairment, each of which accounted for approximately $25 \%$ of these subjects. Among the subjects in the group with MMSE scores $<26$, the combination of executive dysfunction with both attention and memory impairment was most frequent, accounting for approximately $50 \%$ of these subjects. 
The inclusion of impairments in $\geq 2$ cognitive domains as an item in Level I testing stems from the conventional thinking behind the diagnostic criteria for dementia resulting from research on Alzheimer's disease [21]. Level I testing includes 4 cognitive domains that may often be impaired in PD patients. We showed that for the MMSE and MoCA subtests, some test results were abnormal even among PD patients with MMSE scores $\geq 26$. This suggests that for dementia screening tools such as Level I testing, it may be appropriate to use tests in which abnormalities are rare in patients with MMSE scores $\geq 26$ but tend to be common in those scoring $<26$. Thus, once tests that tend to yield abnormal scores even for PD patients with MMSE scores $\geq 26$ are included in a screening tool, its sensitivity for PD-D diagnosis will increase, even if its specificity will decrease.

A total of $32.6 \%$ of the PD patients had MMSE scores $<26$ and impairments in $\geq 2$ cognitive domains, and may therefore have met the diagnostic criteria for probable PD-D by Level I testing [11]. This point prevalence of PD-D in PD is similar to the mean value of $31.1 \%$ (95\% CI: 20.1-42.1) reported in a systematic and critical review of previous studies [22], thus indicating the validity of Level I testing. The fact that $\geq 1$ of the 5 tests concerning the 4 cognitive domains included in these diagnostic tests showed abnormal scores for the group of patients with MMSE scores $<26$ indicates the validity of these tests as a whole for PD-D screening. However, the results of this study show that the MoCA clock drawing subtest might be more appropriate than the phonemic fluency test for revealing executive dysfunction when screening for PD-D. The frequency of abnormal results for the MMSE pentagon copying subtest was $<10 \%$, and thus was markedly lower than for the other tests. This is inconsistent with previous reports [10] which found that visuospatial function tends to be impaired in PD-D, and it may be necessary to replace this test with another that also examines visuospatial function. Our study suggests the MoCA cube copying test may be one candidate.

There are various limitations to our study. Item 4 (activities of daily living) in Level I testing was not evaluated and no extensive neuropsychological assessment was done, because of which dementia was not accurately diagnosed for each subject. Therefore, this study could not address the validity, sensitivity, or specificity of Level I testing regarding clinically diagnosed PD-D. The months reversed test was not used as there was no Japanese equivalent to this English subtest in Level I testing; therefore, we could not examine its validity.

\section{Conclusions}

By using the MMSE and MoCA, Level I testing for PD-D can evaluate both decreased global cognitive efficiency and impairments in cognitive domains that are vulnerable in PD-D. Level I testing is therefore regarded as a practical and useful tool for screening dementia in PD patients. A total of $32.6 \%$ of the PD patients met the diagnostic criteria for probable PD-D. Among the subjects with MMSE scores <26, the combination of impaired attention, executive dysfunction, and memory impairment was the most common (51.4\%). The months reversed test cannot be used in Japanese as a test for attention owing to linguistic nonequivalence. The MoCA clock drawing test may be more appropriate than the phonemic fluency test as a measure of executive function. Perhaps consideration should be given to replacing the MMSE pentagon copying test with a different test of visuospatial function, such as the MoCA cube copying test, to improve sensitivity when screening for PD-D. 
Ohta et al.: Screening Cognitive Domains in Parkinson's Disease

\section{Acknowledgements}

We would like to thank our co-investigators for their contribution: Y. Shinohara and T. Osada, Department of Neurology, Tachikawa Hospital; K. Muramatsu and M. Kunimoto, Department of Neurology, Saiseikai Yokohamashi Tobu Hospital; K. Obara, Department of Neurology, Mito Red Cross Hospital; S. Komatsumoto and K. Isozumi, Department of Neurology, Ashikaga Red Cross Hospital; M. Kobari, Department of Neurology, Shizuoka Red Cross Hospital; B. Mihara, Department of Neurology, Mihara Memorial Hospital; Y. Morita, D. Yasutomi, and K. Gotoh, Department of Neurology, National Hospital Organization Tokyo Medical Center; T. Shirai, Department of Neurology, Eiju General Hospital; Y. Tomita, Department of Neurology, Tomita Hospital; H. Sato and H. Hattori, Department of Neurology, Saitama Municipal Hospital, and H. Nozaki, Department of Neurology, Kawasaki Municipal Kawasaki Hospital.

\section{Disclosure Statement}

None.

\section{References}

$>1$ Chaudhuri KR, Healy DG, Schapira AH: Non-motor symptoms of Parkinson's disease: diagnosis and management. Lancet Neurol 2006;5:235-245.

-2 Schrag A, Jahanshahi M, Quinn N: What contributes to quality of life in patients with Parkinson's disease? J Neurol Neurosurg Psychiatry 2000;69:308-312.

-3 Aarsland D, Larsen JP, Karlsen K, Lim NG, Tandberg E: Mental symptoms in Parkinson's disease are important contributors to caregiver distress. Int J Geriatr Psychiatry 1999;14:866-874.

4 Hely MA, Reid WGJ, Adena MA, Halliday GM, Morris JGL: The Sydney multicenter study of Parkinson's disease: the inevitability of dementia at 20 years. Mov Disord 2008;23:837-844.

5 Armstrong RA, Kotzbauer PT, Perlmutter JS, et al: A quantitative study of $\alpha$-synuclein pathology in fifteen cases of dementia associated with Parkinson disease. J Neural Transm 2014;121:171-181.

6 Shimada H, Hirano S, Shinotoh H, et al: Mapping of brain acetylcholinesterase alterations in Lewy body disease by PET. Neurology 2009;73:273-278.

7 Rowan E, McKeith IG, Saxby BK, et al: Effects of donepezil on central processing speed and attentional measures in Parkinson's disease with dementia and dementia with Lewy bodies. Dement Geriatr Cogn Disord 2007;23: 161-167.

-8 Liepelt I, Maetzler W, Blaicher H-P, Gasser T, Berg D: Treatment of dementia in Parkinsonian syndromes with cholinesterase inhibitors. Dement Geriatr Cogn Disord 2007;23:351-367.

-9 Rolinski M, Fox C, Maidment I, McShane R: Cholinesterase inhibitors for dementia with Lewy bodies, Parkinson's disease dementia and cognitive impairment in Parkinson's disease. Cochrane Database Syst Rev 2012; 3:CD006504.

10 Emre M, Aarsland D, Brown R, et al: Clinical diagnostic criteria for dementia associated with Parkinson's disease. Mov Disord 2007;22:1689-1707.

11 Dubois B, Burn D, Goetz C, et al: Diagnostic procedures for Parkinson's disease dementia: recommendations from the Movement Disorder Society Task Force. Mov Disord 2007;22:2314-2324.

12 Hughes AJ, Daniel SE, Blankson S, Lees AJ: A clinicopathologic study of 100 cases of Parkinson's disease. Arch Neurol 1993;50:140-148.

13 Mori E, Mitani Y, Yamadori A: Usefulness of a Japanese version of the Mini-Mental State Test in neurological patients. Jpn J Neuropsychol 1985;1:82-90.

14 Fujiwara Y, Suzuki H, Yasunaga M, et al: Brief screening tool for mild cognitive impairment in older Japanese: validation of the Japanese version of the Montreal Cognitive Assessment. Geriatr Gerontol Int 2010;10:225232.

15 Ohta K, Osada T, Shinohara Y, et al: Comparing the Montreal Cognitive Assessment with Mini-Mental State Examination in Japanese Parkinson's disease patients. Neurol Clin Neurosci, in press. 
16 Dalrymple-Alford JC, MacAskill MR, Nakas CT, et al: The MoCA: well-suited screen for cognitive impairment in Parkinson disease. Neurology 2010;75:1717-1725.

17 Nasreddine ZS, Phillips NA, Bédirian V, et al: The Montreal Cognitive Assessment, MoCA: a brief screening tool for mild cognitive impairment. J Am Geriatr Soc 2005;53:695-699.

18 Martinez-Martin P, Falup-Pecurariu C, Rodriguez-Blazquez C, et al: Dementia associated with Parkinson's disease: applying the Movement Disorder Society Task Force criteria. Parkinsonism Relat Disord 2011;17: 621-624.

19 di Battista ME, Giustini P, Bernardi S, Stirpe P, Vanacore N, Meco G: A simplified algorithm may lead to overestimate dementia in PD: a clinical and epidemiological study using criteria for PD-D proposed by the Movement Disorders Task Force. J Neural Transm 2011;18:1609-1612.

20 Barton B, Grabli D, Bernard B, et al: Clinical validation of Movement Disorder Society-recommended diagnostic criteria for Parkinson's disease with dementia. Mov Disord 2012;27:248-253.

21 McKhann GM, Knopman DS, Chertkow H, et al: The diagnosis of dementia due to Alzheimer's disease: recommendations from the National Institute on Aging and the Alzheimer's Association workgroup. Alzheimers Dement 2011;7:1-7.

22 Aarsland D, Zaccai J, Brayne C: A systematic review of prevalence studies of dementia in Parkinson's disease. Mov Disord 2005;20:1255-1263.

23 Folstein MF, Folstein SE, McHugh PR: 'Mini-mental state': a practical method for grading the cognitive state of patients for the clinician. J Psychiatr Res 1975;12:189-198. 